Published in final edited form as:

Am Heart J. 2008 January ; 155(1): 82-86.

\title{
Cystatin C and Risk of Heart Failure in the Physicians' Health Study
}

\author{
Luc Djoussé, MD, MPH, DSc ${ }^{1}$, Tobias Kurth, MD, ScD 1,2 , and J. Michael Gaziano, MD, \\ $\mathrm{MPH}^{1,2}$ \\ 1 Division of Aging, Department of Medicine, Brigham and Women's Hospital and Harvard Medical School, \\ Boston, $M A$ \\ 2Division of Preventive Medicine, Department of Medicine, Brigham and Women's Hospital and Harvard \\ Medical School, Boston, MA
}

\section{Abstract}

Background-Chronic kidney disease is a risk factor for heart failure (HF). Though cystatin $\mathrm{C}$ can detect early kidney dysfunction, limited data are available on the association between cystatin $\mathrm{C}$ and HF.

Methods-In a prospective nested case-control study design, we examined whether cystatin C is associated with an increased risk of HF in the Physicians' Health Study and whether such an association is stronger in hypertensive subjects. We selected 220 cases of incident HF and 220 controls, matched on age, year of birth, and time of blood collection. Plasma cystatin C was measured using an immunonephelometry method. We used conditional logistic regression to estimate relative risks.

Results-Compared with the lowest tertile, the multivariable adjusted RR (95\% CI) for HF was 1.15 (0.69-1.89) and 1.78 (1.01-3.13), for the second and third tertile of cystatin C, respectively. Additional adjustment for systolic blood pressure and history of hypertension attenuated this association [RR: 1.0, $1.23(0.73-2.09)$, and $1.61(0.90-2.88)$ from the lowest to the highest tertile, respectively]. Furthermore, we observed a 4-fold increased risk of HF in the second and third tertiles of cystatin $\mathrm{C}$ among hypertensive individuals and no meaningful effects of cystatin $\mathrm{C}$ on $\mathrm{HF}$ among non-hypertensive subjects.

Conclusions-Our data demonstrated that higher levels of cystatin $\mathrm{C}$ are associated with an increased risk of $\mathrm{HF}$ and that such association may be limited to hypertensive individuals. Additional studies are warranted to further examine the relationship between hypertension and cystatin $\mathrm{C}$ on the risk of HF.

\section{Keywords}

Cystatin C; heart failure; epidemiology; risk factors

As a consequence of an increased life expectancy in the US and improved medical treatment, more and more adults are living with chronic conditions, including heart failure (HF). While

Corresponding author: Luc Djoussé, M.D., MPH, D.Sc., Division of Aging, Brigham and Women's Hospital and Harvard Medical School, 1620 Tremont St, $3^{\text {rd }}$ floor; Boston MA 02120, Tel. (617) 525-7591; Fax. (617) 525-7739, E-mail. ldjousse@ rics.bwh.harvard.edu.

Publisher's Disclaimer: This is a PDF file of an unedited manuscript that has been accepted for publication. As a service to our customers we are providing this early version of the manuscript. The manuscript will undergo copyediting, typesetting, and review of the resulting proof before it is published in its final citable form. Please note that during the production process errors may be discovered which could affect the content, and all legal disclaimers that apply to the journal pertain. 
chronic kidney disease has been shown to increase the risk of $\mathrm{HF}^{1-5}$, limited data are available on whether early stages of chronic kidney disease is a risk factor for HF. Cystatin C has been identified as a novel biomarker that is more sensitive to detect early kidney dysfunction compared with creatinine and creatinine-based estimated glomerular filtration rate ${ }^{6-8}$. Unlike other measures of kidney dysfunction, cystatin $\mathrm{C}$ might not be affected by age, gender, and muscle mass ${ }^{9-11}$. Because, cystatin $\mathrm{C}$ is associated with systolic blood pressure ${ }^{12}$, it is important to carefully examine the role of blood pressure on the cystatin C-HF association. Alternatively, elevated serum cystatin C could merely be a reflection of elevated blood pressure and not causally related to HF. In the current study, we sought to evaluate the hypothesis that plasma cystatin $\mathrm{C}$ is positively associated with incident HF among US male physicians and whether the association between cystatin $\mathrm{C}$ and $\mathrm{HF}$ is modified by hypertension.

\section{Methods}

\section{Study population}

The current project is built on the Physicians' Health Study (PHS) I and II. PHS I was a randomized, double blind, placebo-controlled trial designed to test the effects of low-dose aspirin and beta-carotene on cardiovascular disease and cancer among 22,071 US male physicians. PHS II is an ongoing randomized trial designed to test the benefits and risks of vitamins $\mathrm{E}$ and $\mathrm{C}$, beta-carotene, and multivitamin in the prevention of cancer, cardiovascular disease, and age-related eye-diseases and decline in cognitive function among 14,642 US male physicians aged 50 years and older. A detailed description of both studies has been published previously 13,14 . For the current analyses, we used a prospective nested case-control design to select study participants for this pilot project. This pilot study was designed to have $80 \%$ power to detect a $60 \%$ increased risk of HF. Specifically, 220 incident HF cases were randomly selected from a total of $392 \mathrm{HF}$ cases that occurred after a second blood collection (between 1995 and 2001) in the PHS I and II. For each case, we selected one control subject who was alive at the time of the index date of the case and matched on age (within 1 year), year of birth (identical to that of the case), and time of blood collection (same year). Within each risk set, a case and all potential controls were ranked by date of birth and date of blood collection and the nearest control to the case was selected. Each case was eligible to serve as control prior to HF diagnosis. Similarly, each control was eligible to latter become HF case to assure that controls were representative of a total population that gave rise to the HF cases. Each participant gave written informed consent and the Brigham and Women's Hospital Institutional Review Board approved the study protocol.

\section{Blood Collections and Storage}

For the current project, blood was collected between 1995 and 2001. Participants were sent blood collection kits containing: a) 3 EDTA and 3 citrate tubes, b) a gel-filled freezer pack, c) a completed overnight courier air bill, and d) written instructions and other supplies needed for venipuncture 15 . Collected specimens were sent to our laboratory in freezer packs within 24 hours of blood draw. Once received at the laboratory, samples were fractioned into plasma, red blood cells, and buffy coat. The samples were aliquoted into twelve $2 \mathrm{ml}$ Nunc vials (6 EDTA plasma, 3 citrate plasma, 1 EDTA red blood cells, and 1 EDTA and 1 citrate buffy coat), which were frozen at $-170{ }^{\circ} \mathrm{C}$. The entire process was completed swiftly to ensure that samples were frozen within 30 to 36 hours after venipuncture. Samples were received from 17,253 physicians.

\section{Measurement of cystatin C}

Cystatin $\mathrm{C}$ measurement was performed using a particle-enhanced immunonephelometry on the Dade Behring BN 100 System (Dade Behring Inc, Deerfield IL) ${ }^{16}$. In this assay, immune complexes are formed when cystatin $\mathrm{C}$ from the sample is bound to polystyrene particles coated 
with antibody specific to human cystatin C. These immune complexes cause light scattering that is proportional to the concentration of cystatin C. The intraindividual coefficient of variation was $6.9 \%$, which is similar to that of other laboratories $12,17,18$.

\section{Ascertainment of incident heart failure}

A questionnaire was mailed to each participant biennially during the first year and has been mailed annually thereafter to obtain information on the occurrence of major diseases including HF. In a pilot study, we found a higher confirmation rate (90\%) of HF using the Framingham criteria $^{19}$. Detailed description of HF validation has been published elsewhere 20 .

\section{Other variables}

Information on age, height, weight, body mass index, cigarette smoking, vegetable consumption, hypertension, use of multivitamins, atrial fibrillation, valvular disease, diabetes mellitus, and physical activity was collected at baseline through a standardized questionnaire. Incidence of major chronic disease was ascertained through annual follow-up questionnaires and validated through an endpoint committee. High-sensitive C-reactive protein (hs-CRP) was measured using a standard method.

\section{Statistical analyses}

The distribution of cystatin $\mathrm{C}$ was skewed to the right (skewness $=3.3$, kurtosis $=17.9$ ). Thus, we used the natural logarithm to normalize its distribution and created tertiles of cystatin $\mathrm{C}$ based on its distribution in the entire population. We used a conditional logistic regression (where each risk set was considered as a stratum) to compute multivariable-adjusted odds ratios as the measure of the relative risks (RRs) with corresponding 95\% confidence intervals (CIs) using subjects in the lowest tertile as the reference group. We assessed confounding by body mass index (continuous), smoking (yes/no), alcohol intake ( $<1,1-4,5+/$ week), systolic and diastolic blood pressure (continuous), hs-CRP, as well as history of diabetes, myocardial infarction, and hypertension. A variable was considered a confounder if its adjustment led to a $10 \%$ or more change in the relative risk estimates for the cystatin $\mathrm{C}$ (tertiles) and $\mathrm{HF}$ association. We obtained $\mathrm{p}$ value for linear trend by creating a new variable that was assigned the median cystatin $\mathrm{C}$ value in each tertile and then fitting the new variable in a multivariable conditional logistic regression model. The initial model accounted for matching factors only (age, date of blood collection, and year of birth). The parsimonious model adjusted for age, year of birth, body mass index, alcohol intake, smoking, and history of myocardial infarction. Because cystatin $\mathrm{C}$ has been shown to be positively correlated with systolic blood pressure and history of hypertension is a major risk factor for HF, we examined whether the cystatin C-HF association was influenced by blood pressure or hypertension status. Specifically, we included systolic blood pressure (linear) and hypertension status (yes/no) to see whether such adjustment would attenuate the relative risk. Furthermore, we conducted stratified analyses by history of hypertension. In secondary analyses, we used $\log$-transformed cystatin $\mathrm{C}$ as a continuous variable to repeat above analyses. We calculated the $\mathrm{p}$ values for interaction between cystatin $\mathrm{C}$ and hypertension by including a product term in the regression model. All analyses were completed using SAS, version 9.1 (SAS Institute, NC). All P values were two-tailed and we considered an alpha of 0.05 statistically significant.

\section{Results}

Among 220 case-control pairs in the PHS, the mean age (SD) at the 1995-2001 blood collection was 74.1 (8.2) years (range 51.7 to 91.8 years). Table 1 presents baseline characteristics of the study participants. Higher cystatin $\mathrm{C}$ was associated with older age; higher systolic blood pressure; higher prevalence of hypertension, myocardial infarction, and diabetes mellitus; and lower proportion of current drinkers. In a conditional logistic regression controlling for 
matching factors (age, time of blood collection, and year of birth), body mass index, smoking, alcohol use, and history of myocardial infarction, relative risks $(95 \% \mathrm{CI})$ for HF were 1.0 (reference), 1.15 (0.69-1.89), and 1.78 (1.01-3.13) in the first through the third tertile of cystatin $\mathrm{C}$, respectively ( $\mathrm{p}$ for linear trend 0.011 , Table 2). Additional adjustment of systolic blood pressure and history of hypertension attenuated the relative risk with corresponding relative risks of $1.23(0.73-2.09)$ and $1.61(0.90-2.88)$ for the $2^{\text {nd }}$ and $3^{\text {rd }}$ tertile (p for trend 0.11 , Table 2). Cystatin $C$ was significantly and positively correlated with systolic (spearman correlation coefficient $0.13, \mathrm{p}=0.001$ ) but not diastolic blood pressure.

When stratified by hypertension history, cystatin $\mathrm{C}$ was associated with an increased risk of HF among men with hypertension but not in those without hypertension. For hypertensive subjects, the relative risk of HF was four times higher in the second and third tertile of cystatin (Table 3) after adjusting for potential confounders ( $\mathrm{p}$ for interaction 0.35 ). This positive association among hypertensive subjects persisted even after additional adjustment for hs-CRP (RR: 1.0, $3.40(0.90-13.60), 3.70(1.10-13.10)$ from the lowest to the highest teritle of cystatin $\mathrm{C}$, respectively, $\mathrm{p}$ for trend 0.04 ). Lastly, analyzed as a continuous variable, log-transformed cystatin $\mathrm{C}$ was significantly associated with an increased risk of HF in the total population (Table 4). This association was attenuated but remained statistically significant after additional adjustment for systolic blood pressure and hypertension. However, there was a positive but not statistically significant association between log-cystatin $\mathrm{C}$ and $\mathrm{HF}$ in hypertensive subjects; no substantial association between cystatin $\mathrm{C}$ and $\mathrm{HF}$ among non-hypertensive subjects ( $\mathrm{p}$ for interaction $=0.29$, Table 4 ).

\section{Discussion}

In the current prospective nested case-control study, we demonstrated that higher levels of serum cystatin $\mathrm{C}$ were associated with an increased risk of $\mathrm{HF}$ and that such association was attenuated by further adjustment for blood pressure and hypertension status. When stratified by hypertension status, there was no statistically significant association between cystatin $\mathrm{C}$ and $\mathrm{HF}$ among non-hypertensive subjects, whereas a strong positive relation was seen between cystatin $\mathrm{C}$ and $\mathrm{HF}$ among hypertensive individuals.

These findings are consistent with previous reports on cystatin C and HF. Data from the Cardiovascular Health Study showed a stepwise increased risk of HF with cystatin C (2.2-fold increased risk of $\mathrm{HF}$ comparing the fifth to the first quintile of cystatin C) among participants aged 65 years and older at baseline ${ }^{4}$. Among older adults without chronic kidney disease, cystatin $\mathrm{C}$ was associated with a $28 \%$ increased risk of $\mathrm{HF}^{5}$. Furthermore, data from the Heart and Soul Study have demonstrated that being in the fourth quartile of cystatin $\mathrm{C}$ was associated with a 2.6-fold increased risk of HF compared with the first quartile of cystatin C among 990 individuals with coronary heart disease ${ }^{21}$. In the Health, Aging, and Body Composition (ABC) Study ${ }^{18}$, cystatin $\mathrm{C}$ was positively associated with the risk of $\mathrm{HF}$ in a dose-response fashion in black participants, with a relative risk (95\% CI) of 3.0 (1.4-6.5) comparing the fifth with the first quintile of cystatin $\mathrm{C}$; in contrast, the relative risk of $\mathrm{HF}$ was close to unity in the first through the fourth quintile in white subjects of the same study with a modest and nonstatistically significant increased risk in the fifth quintile $[R R=1.4(0.8-2.5)]$. Our data derived from a predominantly white population are not completely consistent with the Health $\mathrm{ABC}$ Study ${ }^{18}$, though the overlapping confidence intervals point to certain degree of agreement. Several factors could account for this discrepancy including small sample size, shorter followup time, and advanced age among participants of the Health ABC Study.

Contrary to our study, previous studies have evaluated the influence of hypertension by including hypertension variable in the regression model. If extreme categories of cystatin C have only limited number of subjects with hypertension, such analytical method may be 
inadequate in determining whether the association between cystatin $\mathrm{C}$ and $\mathrm{HF}$ is independent of hypertension. Hypertension is a strong risk factor for $\mathrm{HF}^{22-24}$. Since cystatin $\mathrm{C}$ is a biomarker with a better sensitivity (than creatinine-based estimated glomerular filtration rate) to detect early kidney dysfunction 7,8 , it is possible that it could be a surrogate of systolic blood pressure and not be an independent predictor of HF. If the later hypothesis were true, then one would expect a strong correlation between cystatin $\mathrm{C}$ and systolic blood pressure. In our study, we observed a weak spearman correlation coefficient between cystatin $\mathrm{C}$ and systolic blood pressure ( $r=0.13, p=0.001$ ), thus lending only a modest support to this theory. Data from the Heart and Soul Study have shown a linear relation between cystatin C and systolic blood pressure and pulse pressure among 906 participants with prevalent coronary heart disease 12 . In addition, cystatin $\mathrm{C}$ has been positively related to left ventricular hypertrophy and left ventricular diastolic dysfunction 25,26 .

On the other hand, the association between cystatin $\mathrm{C}$ and HF may be mediated through other mechanisms. Cystatin $\mathrm{C}$ is an inhibitor of cysteine proteases of the cathepsin family (including elastases and collagenases $)^{27}$ that are known to play a role in atherosclerosis 28 . Cysteine proteases degrade elastin and collagen - two important constituents of extracellular matrix of vascular walls ${ }^{29}$. It is therefore possible that cystatin C plays a role in matrix remodeling, a process involved in HF development. However, experiments in animals have not been consistent with the hypothesis that elevated concentrations of cystatin $\mathrm{C}$ are associated with cardiovascular disease and HF. Apolipoprotein E-deficient mice lacking cystatin $\mathrm{C}$ showed larger atherosclerotic plaques than those with cystatin $\mathrm{C}$ after several weeks of intervention with an atherogenic diet ${ }^{30,31}$, suggesting protective role of cystatin $\mathrm{C}$. This apparent cystatin $\mathrm{C}$ paradox could be partially explained by the involvement of inflammation among subjects with elevated cystatin $\mathrm{C}$ concentrations. Raised cystatin $\mathrm{C}$ may be associated with microinflammation, which plays an important role in the development of atherosclerosis. Since, all nucleated cells secrete cystatin $\mathrm{C}$, its serum concentration is not affected by its deficiency in atherosclerotic lesions. The fact that our point estimate was modestly attenuated upon further adjustment of hs-CRP lends support to this theory.

Our study has several limitations. First, we did not collect data to distinguish HF with and without preserved left ventricular function. Since cystatin $\mathrm{C}$ is positively associated with systolic blood pressure, however, we would anticipate a stronger relation between cystatin C and diastolic heart failure as a consequence of hypertensive cardiomyopathy. We are currently collecting data to test this hypothesis in the PHS. Second, we had limited power to detect an interaction between hypertension and cystatin $\mathrm{C}$ on HF in this pilot study. However, based on the qualitatively different association between cystatin $\mathrm{C}$ and $\mathrm{HF}$ according the hypertension status, we would expect statistically significant interaction in larger studies. Third, our sample consists of highly educated male physicians who may have different behaviors than the general population; thus limiting the generalizability of our findings. Fourth, our study is an observational study and we cannot exclude residual or unmeasured confounding as possible alternative explanation of our results. Lastly, we did not have data on microalbuminuria for further evaluation. On the other hand, this study has numerous strengths, including matching on key confounders such as age and year of birth to minimize confounding; the ability to control for residual confounding through collected covariates; the prospective design; the high confirmation rate of self-reported HF using the Framingham criteria; and the longer follow-up time.

In conclusion, our data show a positive association between cystatin $\mathrm{C}$ and HF. The attenuation of this association by further adjustment of blood pressure and the fact that it was restricted to hypertensive subjects raises the question whether cystatin $\mathrm{C}$ is an independent risk factor for HF or just a biomarker for earlier blood pressure effects on HF. Further exploration of the 
physiologic mechanisms and connections between cystatin $\mathrm{C}$ and blood pressure on HF are warranted.

\section{Acknowledgements}

We are indebted to the participants in the PHS for their outstanding commitment and cooperation and to the entire PHS staff for their expert and unfailing assistance.

Funding: The Physicians' Health Study is supported by grants CA-34944 and CA-40360 from the National Cancer Institute and grants HL-26490 and HL-34595 from the National Heart, Lung, and Blood Institute, Bethesda, MD. Dr. Djoussé is Principal Investigator on a K01 HL-70444 from the National Heart, Lung, and Blood Institute, Bethesda, MD.

\section{References}

1. Gottdiener JS, Arnold AM, Aurigemma GP, et al. Predictors of congestive heart failure in the elderly: the Cardiovascular Health Study. J Am Coll Cardiol 2000;35:1628-1637. [PubMed: 10807470]

2. Chae CU, Albert CM, Glynn RJ, et al. Mild renal insufficiency and risk of congestive heart failure in men and women > or =70 years of age. Am J Cardiol 2003;92:682-686. [PubMed: 12972106]

3. Bibbins-Domingo K, Lin F, Vittinghoff E, et al. Predictors of heart failure among women with coronary disease. Circulation 2004;110:1424-1430. [PubMed: 15353499]

4. Sarnak MJ, Katz R, Stehman-Breen CO, et al. Cystatin C concentration as a risk factor for heart failure in older adults. Ann Intern Med 2005;142:497-505. [PubMed: 15809461]

5. Shlipak MG, Katz R, Sarnak MJ, et al. Cystatin C and prognosis for cardiovascular and kidney outcomes in elderly persons without chronic kidney disease. Ann Intern Med 2006;145:237-246. [PubMed: 16908914]

6. Bokenkamp A, Domanetzki M, Zinck R, et al. Cystatin C--a new marker of glomerular filtration rate in children independent of age and height. Pediatrics 1998;101:875-881. [PubMed: 9565418]

7. Newman DJ, Thakkar H, Edwards RG, et al. Serum cystatin C measured by automated immunoassay: a more sensitive marker of changes in GFR than serum creatinine. Kidney Int 1995;47:312-318. [PubMed: 7731163]

8. Fliser D, Ritz E. Serum cystatin C concentration as a marker of renal dysfunction in the elderly. Am J Kidney Dis 2001;37:79-83. [PubMed: 11136171]

9. Keevil BG, Kilpatrick ES, Nichols SP, et al. Biological variation of cystatin C: implications for the assessment of glomerular filtration rate. Clin Chem 1998;44:1535-1539. [PubMed: 9665434]

10. Randers E, Erlandsen EJ. Serum cystatin $\mathrm{C}$ as an endogenous marker of the renal function--a review. Clin Chem Lab Med 1999;37:389-395. [PubMed: 10369108]

11. Coll E, Botey A, Alvarez L, et al. Serum cystatin C as a new marker for noninvasive estimation of glomerular filtration rate and as a marker for early renal impairment. Am J Kidney Dis 2000;36:2934. [PubMed: 10873868]

12. Peralta CA, Whooley MA, Ix JH, et al. Kidney function and systolic blood pressure new insights from cystatin C: data from the Heart and Soul Study. Am J Hypertens 2006;19:939-946. [PubMed: 16942937]

13. Final report on the aspirin component of the ongoing Physicians' Health Study. Steering Committee of the Physicians' Health Study Research Group. N Engl J Med 1989;321:129-135. [PubMed: 2664509]

14. Christen WG, Gaziano JM, Hennekens CH. Design of Physicians' Health Study II--a randomized trial of beta-carotene, vitamins $\mathrm{E}$ and $\mathrm{C}$, and multivitamins, in prevention of cancer, cardiovascular disease, and eye disease, and review of results of completed trials. Ann Epidemiol 2000;10:125-134. [PubMed: 10691066]

15. Rexrode KM, Buring JE, Glynn RJ, et al. Analgesic use and renal function in men. JAMA 2001;286:315-321. [PubMed: 11466097]

16. Wasen E, Isoaho R, Mattila K, et al. Estimation of glomerular filtration rate in the elderly: a comparison of creatinine-based formulae with serum cystatin C. J Intern Med 2004;256:70-78. [PubMed: 15189368] 
17. Watanabe S, Okura T, Liu J, et al. Serum cystatin C level is a marker of end-organ damage in patients with essential hypertension. Hypertens Res 2003;26:895-899. [PubMed: 14714581]

18. Bibbins-Domingo K, Chertow GM, Fried LF, et al. Renal function and heart failure risk in older black and white individuals: the Health, Aging, and Body Composition Study. Arch Intern Med 2006;166:1396-1402. [PubMed: 16832005]

19. Ho KK, Anderson KM, Kannel WB, et al. Survival after the onset of congestive heart failure in Framingham Heart Study subjects. Circulation 1993;88:107-115. [PubMed: 8319323]

20. Djousse L, Gaziano JM. Alcohol consumption and risk of heart failure in the Physicians' Health Study I. Circulation 2007;115:34-39. [PubMed: 17130341]

21. Ix JH, Shlipak MG, Chertow GM, et al. Association of cystatin C with mortality, cardiovascular events, and incident heart failure among persons with coronary heart disease: data from the Heart and Soul Study. Circulation 2007;115:173-179. [PubMed: 17190862]

22. Kannel WB, Cupples AC. Epidemiology and risk profiles of cardiac failure. Cardiovascular Drugs and Therapy 1988;2:387-395. [PubMed: 3154646]

23. Lloyd-Jones DM. The risk of congestive heart failure: sobering lessons from the Framingham Heart Study. Curr Cardiol Rep 2001;3:184-190. [PubMed: 11305971]

24. Kannel WB. Incidence and epidemiology of heart failure. Heart Fail Rev 2000;5:167-173. [PubMed: 16228142]

25. Ix JH, Shlipak MG, Chertow GM, et al. Cystatin C, left ventricular hypertrophy, and diastolic dysfunction: data from the Heart and Soul Study. J Card Fail 2006;12:601-607. [PubMed: 17045178]

26. Mitsnefes M, Kimbal T, Kartal J, et al. Serum cystatin C and left ventricular diastolic dysfunction in children with chronic kidney disease. Pediatr Nephrol 2006;21:1293-1298. [PubMed: 16721586]

27. Abrahamson M, Alvarez-Fernandez M, Nathanson CM. Cystatins. Biochem Soc Symp 2003:179199. [PubMed: 14587292]

28. Liu J, Sukhova GK, Sun JS, et al. Lysosomal cysteine proteases in atherosclerosis. Arterioscler Thromb Vasc Biol 2004;24:1359-1366. [PubMed: 15178558]

29. Chapman HA, Riese RJ, Shi GP. Emerging roles for cysteine proteases in human biology. Annu Rev Physiol 1997;59:63-88. [PubMed: 9074757]

30. Bengtsson E, To F, Hakansson K, et al. Lack of the cysteine protease inhibitor cystatin C promotes atherosclerosis in apolipoprotein E-deficient mice. Arterioscler Thromb Vasc Biol 2005;25:21512156. [PubMed: 16051881]

31. Bengtsson E, To F, Grubb A, et al. Absence of the protease inhibitor cystatin C in inflammatory cells results in larger plaque area in plaque regression of apoE-deficient mice. Atherosclerosis 2005;180:45-53. [PubMed: 15823274] 


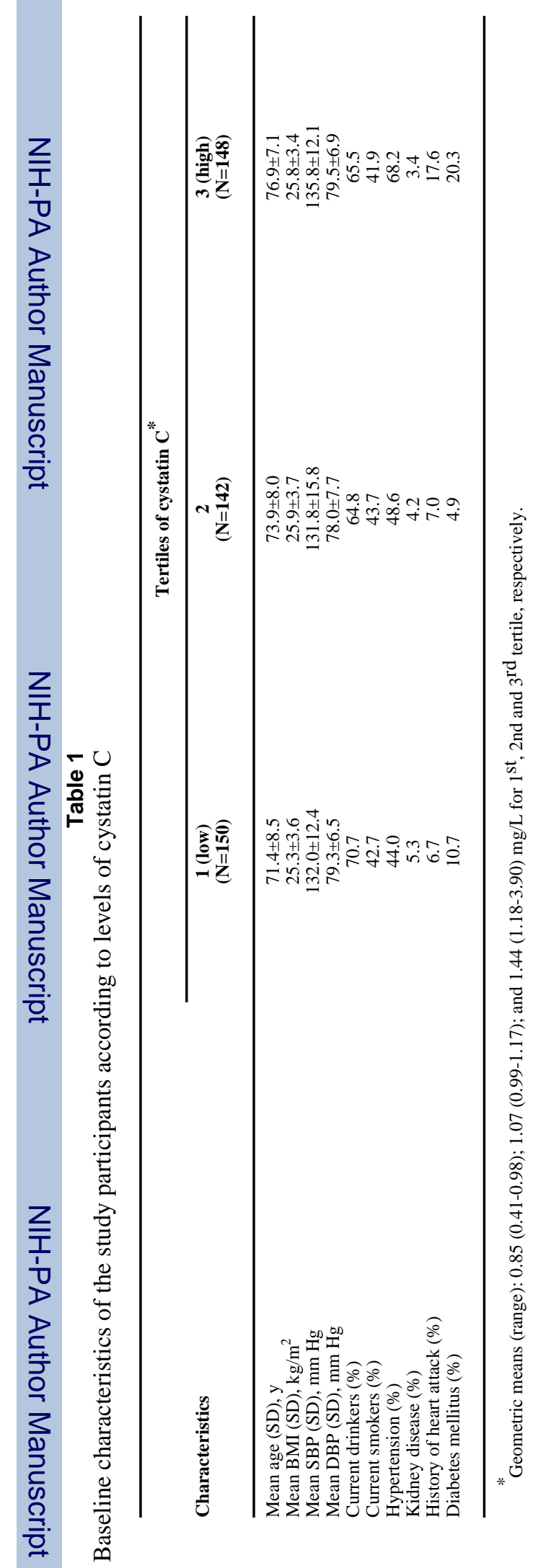




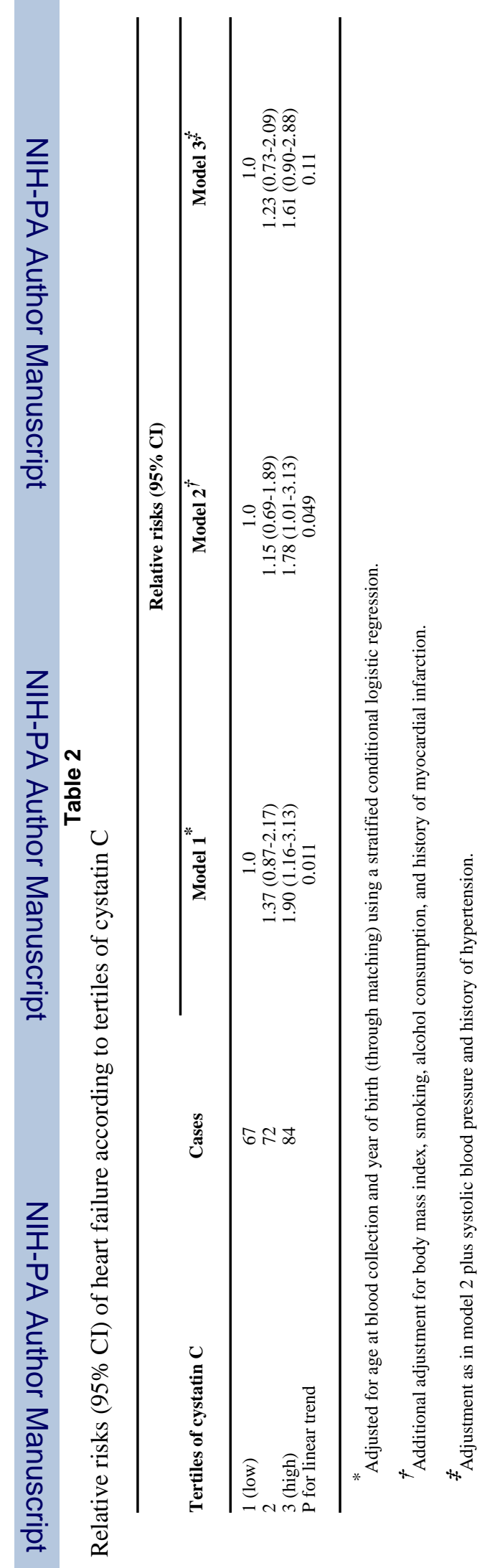




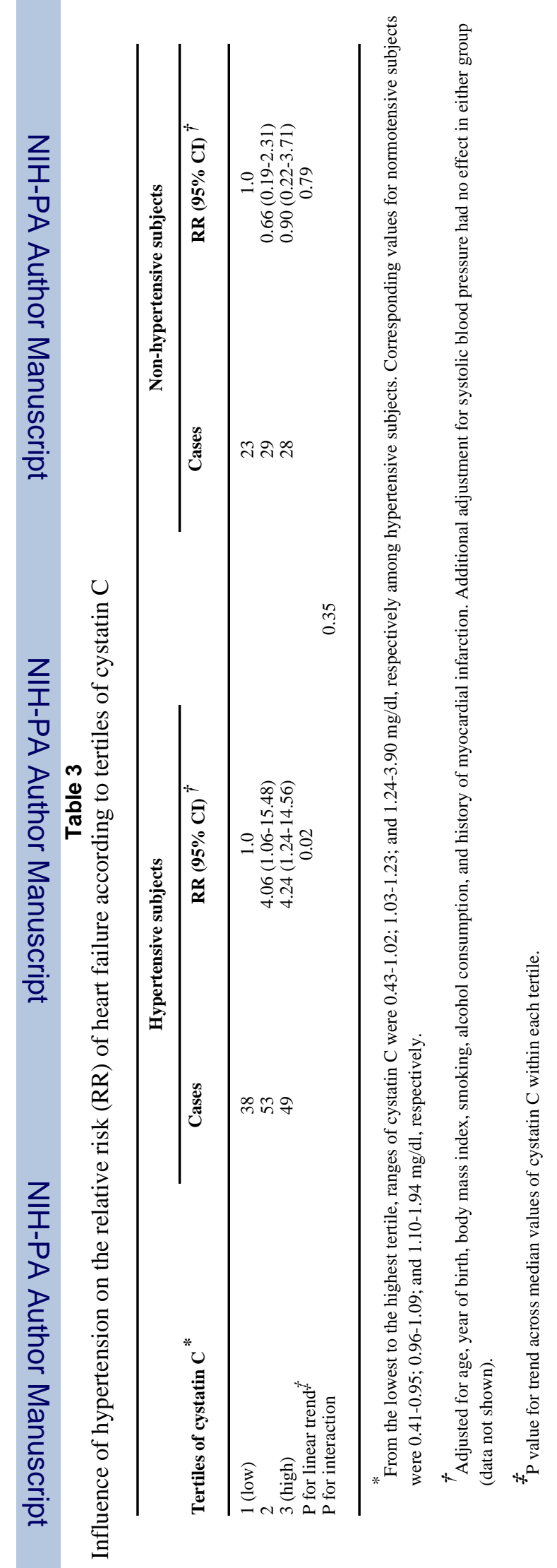

Am Heart J. Author manuscript; available in PMC 2009 January 1. 


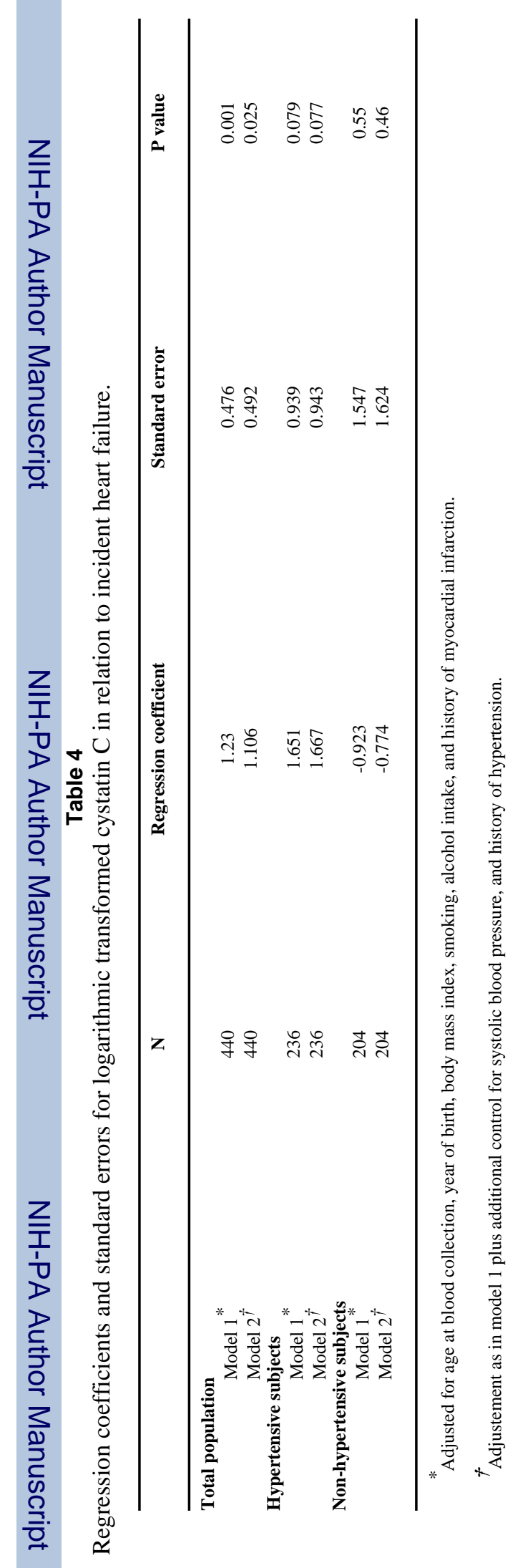

